\title{
The role of magnetic resonance cholangiopancreatography and diffusion-weighted imaging for the differential diagnosis of obstructive biliary disorders
}

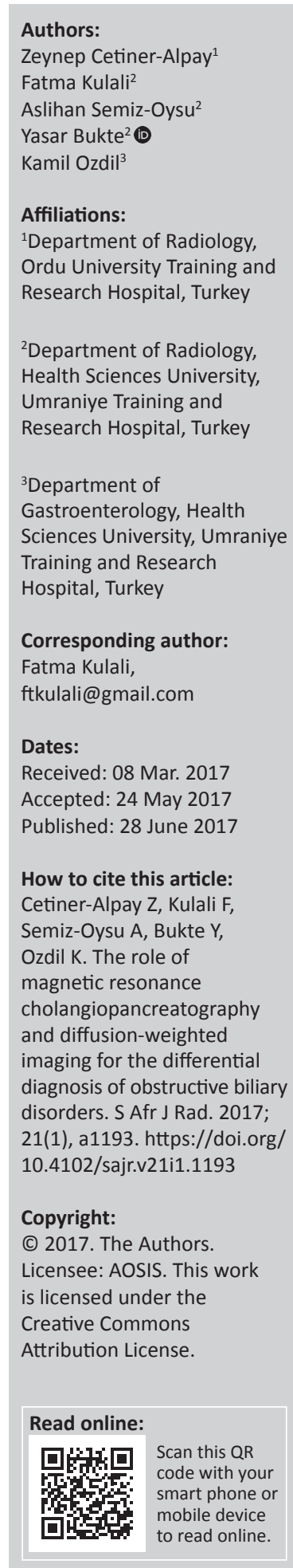

Background: Although endoscopic retrograde cholangiopancreatography (ERCP) is accepted as the gold standard, there is a place for magnetic resonance cholangiopancreatography (MRCP) and diffusion-weighted imaging (DWI) in the diagnosis of obstructive biliary disorders.

Aim: To compare the findings of MRCP with ERCP in patients with obstructive biliary disorders and to investigate the diagnostic efficacy of MRCP combined with DWI.

Study design: Retrospective, analytic, cross-sectional study.

Methods: The MRCP images of 126 patients who underwent both MRCP and ERCP owing to biliary obstruction were reviewed. Nine patients were excluded because of incomplete diagnostic workup or a long period ( $>3$ months) between MRCP and ERCP. Ninety-two patients underwent DWI, which was also evaluated. The sensitivity, specificity and accuracy of MRCP and DWI were analysed.

Results: The sensitivity, specificity and accuracy of MRCP according to ERCP results as the gold standard was $97 \%, 71 \%$ and $93 \%$ for assessment of biliary dilatation; $100 \%, 94.7 \%$ and $97.5 \%$ for the diagnosis of choledocholithiasis; $93.7 \%, 100 \%$ and $99 \%$ for the identification of benign strictures; $100 \%, 100 \%$ and $100 \%$ for the diagnosis of malignant tumours; and $100 \%$, $100 \%$ and $100 \%$ for the detection of complicated hydatid cysts; respectively. The sensitivity and specificity of DWI for the diagnosis of malignant tumour was $100 \%$. In the detection of choledocholithiasis, the sensitivity and specificity of DWI was $70.8 \%$ and $100 \%$.

Conclusions: MRCP is an alternative, non-invasive, diagnostic modality, comparable with ERCP for the evaluation of pancreaticobiliary diseases. DWI can be helpful for diagnosis of choledocholithiasis and tumours.

\section{Introduction}

Biliary obstruction may be related to various aetiologies such as calculus, stricture, trauma, infection or tumour, the most common being choledocholithiasis. An accurate diagnosis is necessary for treatment planning.

Endoscopic retrograde cholangiopancreatography (ERCP) is the gold standard for visualisation of pancreaticobiliary ducts and provides an opportunity for interventional treatment procedures in the same setting. ${ }^{1,2}$ The disadvantages of ERCP are that it is an invasive procedure, the patient is at risk for complications, the study involves radiation, contrast material is necessary and there is a requirement for sedation. Owing to these disadvantages, ERCP is preferred for therapeutic intervention whilst magnetic resonance cholangiopancreatography (MRCP) has gained wide acceptance for diagnostic evaluation. ${ }^{3,4}$

Abdominal magnetic resonance imaging (MRI) and MRCP are non-invasive modalities that allow multiplanar evaluation with reformatting in the coronal and sagittal planes and three-dimensional (3D) reconstruction to provide detailed anatomical information. MRCP is favourable because there is a lack of radiation, contrast material is not required and it can be safely performed in patients with renal insufficiency or contrast allergy. MRCP is the best choice in patients who have contraindications for ERCP, such as patients with allergy to iodinated contrast material and those who cannot receive anaesthesia. ${ }^{5,6} \mathrm{MRCP}$ can prevent unnecessary ERCP interventions and, in 
certain cases, mortality and morbidity owing to ERCP can be avoided. The aims of our study were to analyse the diagnostic effectiveness of MRCP compared with ERCP and to evaluate the role of diffusion-weighted imaging (DWI) in obstructive biliary disease.

\section{Materials and methods}

The Institutional Review Board approved the present retrospective, cross-sectional, analytic study, and informed consent was waived. Between January 2013 and December 2013 , the patients $(N=126)$ who underwent MRCP and then ERCP for suspected biliary obstruction were reviewed via the hospital's picture archiving and communications system (PACS). Six cases with incomplete diagnostic workup were excluded. Three cases were also eliminated because of the long period ( $>3$ months) between MRCP and ERCP. A total of 117 patients were enrolled in the present study. The findings on MRCP and, if available, DWI, were analysed. An experienced radiologist evaluated the MRCP and DWI findings without knowledge of the ERCP findings. The presence of calculus, biliary dilatation, malignant tumour, stricture and hydatid cyst communicating with the biliary system was evaluated. The largest diameter $(<10 \mathrm{~mm} / \geq 10$ $\mathrm{mm}$ ) and number of stones (solitary/multiple) were also noted. Apparent diffusion coefficient (ADC) values of tumours were calculated on a workstation (Leonardo, Siemens Medical Solutions, Erlangen, Germany).

All MRCPs were performed on a 1.5 Tesla system (Magnetom Avanto, Siemens Medical Solutions), using a phased array body coil. Routine MRCP sequences were performed. The common bile duct was localised on coronal T2 half-Fourieracquisition single-shot turbo spin-echo (HASTE) (TR/TE 1000/130, field of view (FOV) 420, slice thickness $7 \mathrm{~mm}$, matrix $216 \times 320$ ) sequence. Axial T2 HASTE and fatsaturated T2- (TR/TE 800/162, FOV 440, flip angle $180^{\circ}$, slice thickness $4 \mathrm{~mm}$ ) weighted images were achieved. Coronal thin slice $(4 \mathrm{~mm})$ and thick slab $(7 \mathrm{~mm}) \quad 3 \mathrm{D}$ respiratory-triggered heavily $\mathrm{T} 2$-weighted fast spin-echo (FSE) (TR/TE1580/594, FOV420, matrix 382×384) sequences were performed, focusing on the common bile duct. Reconstruction was done by using the Siemens-Advantage Windows programme to achieve MRCP images. Upper abdominal DWI was done by single-shot echo-planar imaging sequence (TR4200, TE83, FOV440, NEX2, matrix $144 \times 192$, slice thickness $6 \mathrm{~mm}$ ) with $b=0, b=400, b=800 \mathrm{~s}$ / $\mathrm{mm}^{2}$ values.

A Fujinon ED530-XTduodenoscope with a side-view endoscope was used for ERCP by an experienced gastroenterologist. After cannulation of the ampulla of Vater, contrast was administered to illustrate the biliary system. During ERCP, procedures such as sphincterotomy, balloon dilatation, stone extraction, stent placement or biopsy were performed, where appropriate.

The sensitivity, specificity, positive predictive value (PPV), negative predictive value (NPV) and accuracy of MRCP for diagnosis of the aetiology of obstructive biliary disease were estimated. The sensitivity, specificity, PPV, NPV and accuracy of DWI for diagnosis of choledocholithiasis, and malignant and benign strictures were calculated. Confidence intervals (CIs) for sensitivity and specificity are 'exact' ClopperPearson CIs (CI 95\%).

\section{Ethical considerations}

Ethics committee approval was received for the study from the Ethics Committee of Health Sciences University Umraniye Training and Research Hospital (date of approval 14 January 2014; ref. number 2014/727).

\section{Results}

A total of 117 patients (66 (56.4\%) female and 51 (43.6\%) male) were included in our study. Their mean age was 69 years (range 16-89 years). DWI was performed on 92 (78.6\%) patients. The distribution of findings is shown in Table 1. The sensitivity, specificity and accuracy of MRCP for the detection of biliary dilatation compared with ERCP were 97\%, 71\% and $93 \%$, respectively.

\section{Choledocholithiasis}

The stones at ERCP and MRCP were evaluated according to their number as solitary or multiple $(\geq 2)$, and largest diameter as $<10 \mathrm{~mm}$ and $\geq 10 \mathrm{~mm}$ (Figure 1). Amongst stones $<10 \mathrm{~mm}$, there were three false positive results at MRCP. All 33 patients with stones $\geq 10 \mathrm{~mm}$ at ERCP were also diagnosed at MRCP. There were 35 patients with a solitary stone and 31 patients with multiple stones at MRCP. There were two false negative results for solitary stones and five false positive results for multiple stones on estimation of stone number. The distribution of stones is shown in Table 2.

During ERCP, calculi were extracted in 57 of 63 patients. In the remaining six patients, the stones were very small and passed out spontaneously. With ERCP as the gold standard, the sensitivity, specificity, PPV, NPV and accuracy of MRCP for diagnosis of choledocholithiasis was 100\%, 94.7\%, 95.4\%, $100 \%$ and $97.5 \%$, respectively.

Amongst the 63 patients with choledocholithiasis at ERCP, $48(76.2 \%)$ patients also underwent DWI. However,

TABLE 1: The distribution of findings in 117 patients with obstructive biliary disorders on both endoscopic retrograde cholangiopancreatography and magnetic resonance cholangiopancreatography.

\begin{tabular}{lcccccc}
\hline Findings & \multicolumn{2}{c}{ ERCP } & & \multicolumn{2}{c}{ MRCP } \\
\cline { 2 - 3 } \cline { 6 - 7 } & $\boldsymbol{n}$ & $\mathbf{\%}$ & & $\boldsymbol{n}$ & $\%$ \\
\hline Normal & 17 & 14.5 & & 15 & 12.8 \\
Choledocholiathiasis & 63 & 53.8 & & 66 & 56.4 \\
Tumour & 18 & 15.4 & & 18 & 15.4 \\
Benign stricture & 16 & 13.7 & & 15 & 12.8 \\
Ruptured hydatid cyst & 3 & 2.6 & & 3 & 2.6 \\
\hline
\end{tabular}

ERCP, endoscopic retrograde cholangiopancreatography; MRCP, magnetic resonance cholangiopancreatography. 

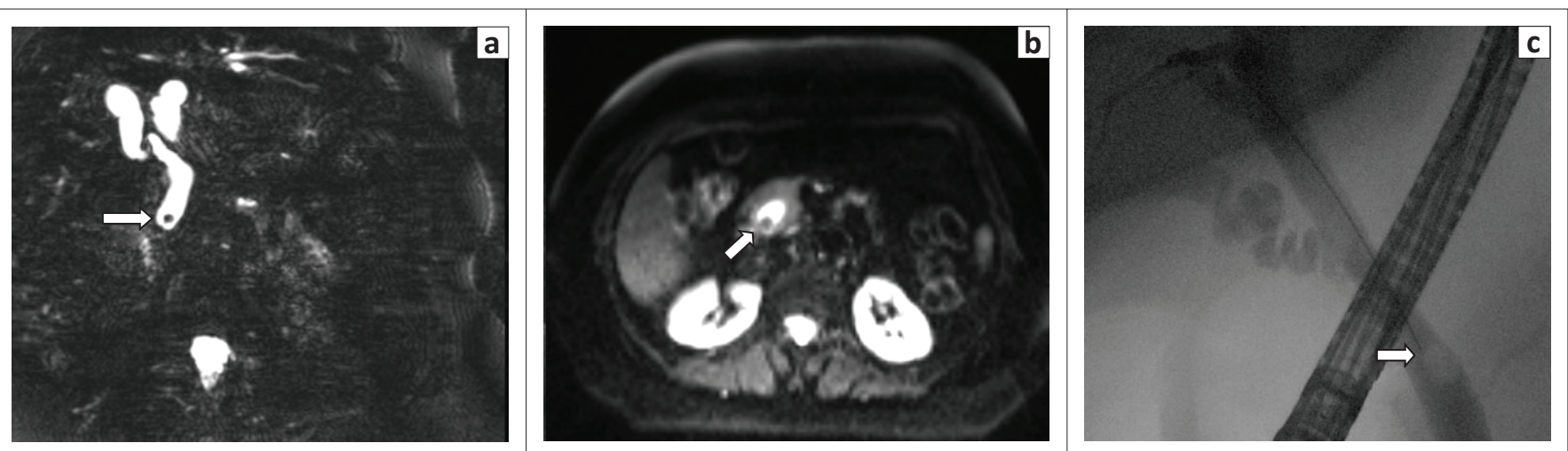

MRCP, magnetic resonance cholangiopancreatography; ERCP, endoscopic retrograde cholangiopancreatography.

FIGURE 1: Seventy-year-old man with $<10 \mathrm{~mm}$ choledocholithiasis (arrow) as imaged by (a) MRCP and (b) DWI, and (c) ERCP demonstrating a subcentimeter stone (arrow).

TABLE 2: The distribution of stones according to the number and diameter of stones on endoscopic retrograde cholangiopancreatography and magnetic resonance cholangiopancreatography.

\begin{tabular}{lcc}
\hline Stones & ERCP $(\boldsymbol{n}=\mathbf{6 3})$ & MRCP $(\boldsymbol{n}=\mathbf{6 6})$ \\
\hline$<10 \mathrm{~mm}$ solitary & 21 & 19 \\
$<10 \mathrm{~mm}$ multiple & 9 & 14 \\
$\geq 10 \mathrm{~mm}$ solitary & 16 & 16 \\
$\geq 10 \mathrm{~mm}$ multiple & 17 & 17 \\
\hline
\end{tabular}

ERCP, endoscopic retrograde cholangiopancreatography; MRCP, magnetic resonance cholangiopancreatography.

choledocholithiasis was only seen in 34 patients on $b=0$ images. In the detection of choledocholithiasis, the sensitivity, specificity, PPV, NPV and accuracy of DWI was 70.8\% (95\%CI $55.94 \%-83.05 \%), 100 \%$ (95\% CI 91.96\%-100\%), 100\% (95\%CI $89.84 \%-100 \%), 79.4 \%$ (95\%CI $66.91 \%-83.01 \%$ ) and $86.2 \%$, respectively.

\section{Malignant tumours}

Both MRCP and ERCP demonstrated a tumour as the cause of biliary obstruction in 18 patients. Amongst these, four patients had pancreatic adenocarcinoma, six patients had cholangiocarcinoma, two patients had ampullary adenocarcinoma, one patient had pancreatic neuroendocrine tumour, and one patient had gallbladder adenocarcinoma infiltrating into the biliary bifurcation. Four patients refused biopsy or surgery, and therefore no histological diagnoses were obtained. All malignant strictures owing to tumour were accurately detected with MRCP (Figure 2). The sensitivity and specificity of MRCP in the diagnosis of malignant tumour was $100 \%$.

Fourteen patients with malignant tumours also had DWI. All tumours showed diffusion restriction, and DWI was useful in the diagnosis of malignant tumours. The mean ADC value of malignant tumours was $1.306 \pm 0.30$ $\times 10^{-3} \mathrm{~mm}^{2} / \mathrm{s}$ (range $0.9-1.9 \times 10^{-3} \mathrm{~mm}^{2} / \mathrm{s}$ ). Lower ADC values were calculated in high-grade adenocarcinomas. Some malignant tumours with a cystic component $(n=3)$ showed higher ADC values. The sensitivity, specificity, PPV, NPV and accuracy of DWI for diagnosis of malignant tumour was $100 \%(95 \%$ CI $76.84 \%-100.00 \%), 100 \%$ (95\%CI $96.48 \%-100.00 \%), 100 \%, 100 \%$ and $100 \%$, respectively.

\section{Benign strictures}

The final diagnosis of 16 benign strictures was made by combined assessment of ERCP and clinical and laboratory data. Of these, 15 cases were diagnosed as having a benign stricture at MRCP (Figure 3). The remaining one case was misdiagnosed as a subcentimeter stone on MRCP. The sensitivity, specificity, PPV, NPV and accuracy of MRCP in the diagnosis of benign strictures was $93.7 \%, 100 \%, 100 \%$, $99 \%$, and $99 \%$, respectively.

\section{Hydatid cysts}

There were three hydatid cysts that had ruptured into the biliary system. The scolexes and membranes were extracted from the biliary system at ERCP. All ruptured hydatid cysts were detected at MRCP (Figure 4). Despite the small sample size, all the sensitivity, specificity, PPV and NPV values of MRCP in the detection of ruptured hydatid cyst were $100 \%$.

The statistical analyses of MRCP findings compared with ERCP results as the gold standard are summarised in Table 3.

\section{Discussion}

MRCP is a non-invasive modality for investigating the morphological features of the pancreaticobiliary system. ${ }^{5,7}$ When T2-weighted sequences and MRCP images are evaluated together, detailed information can be obtained in the evaluation of extraductal causes of biliary obstruction, which provides an important advantage of MRCP over ERCP. ${ }^{8}$ MRCP has no known side effects, although it is contraindicated in patients with cardiac pacemakers, cochlear implants and other metal implants. In addition, there is no need for contrast and sedation with MRCP, as opposed to ERCP. Despite the risk of complications, ERCP is generally accepted as the gold standard because it allows demonstration of the biliary ducts as well as the performance of therapeutic interventions. ${ }^{1,2}$

A number of studies have compared MRCP with ERCP findings. Ito et al. ${ }^{9}$ evaluated the clinical efficacy of MRCP in the diagnosis of biliary tract disease in comparison with ERCP in 311 patients. The sensitivity of MRCP for diagnosis 


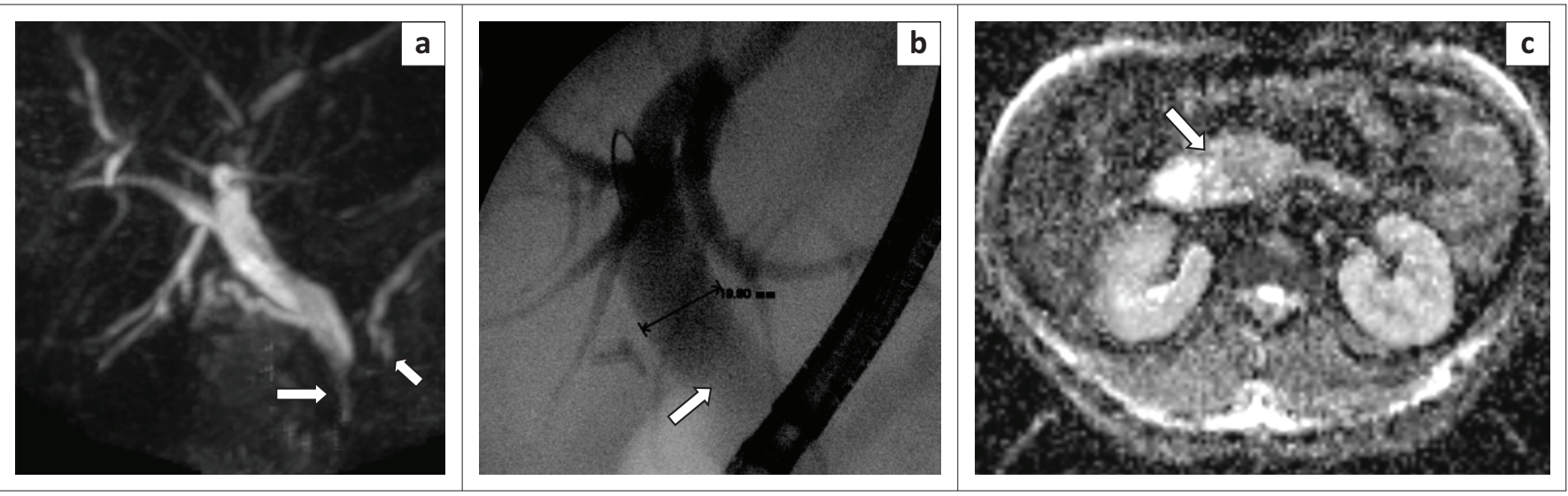

MRCP, magnetic resonance cholangiopancreatography; ERCP, endoscopic retrograde cholangiopancreatography

FIGURE 2: Forty-four-year-old woman with adenocarcinoma of the pancreas: (a) The double duct sign (arrows) is seen on MRCP image; (b) abrupt termination of the dilated common bile duct (arrow) is shown in ERCP image; (c) on the apparent diffusion coefficient image, there is a hypointense signal owing to diffusion restriction (arrow).
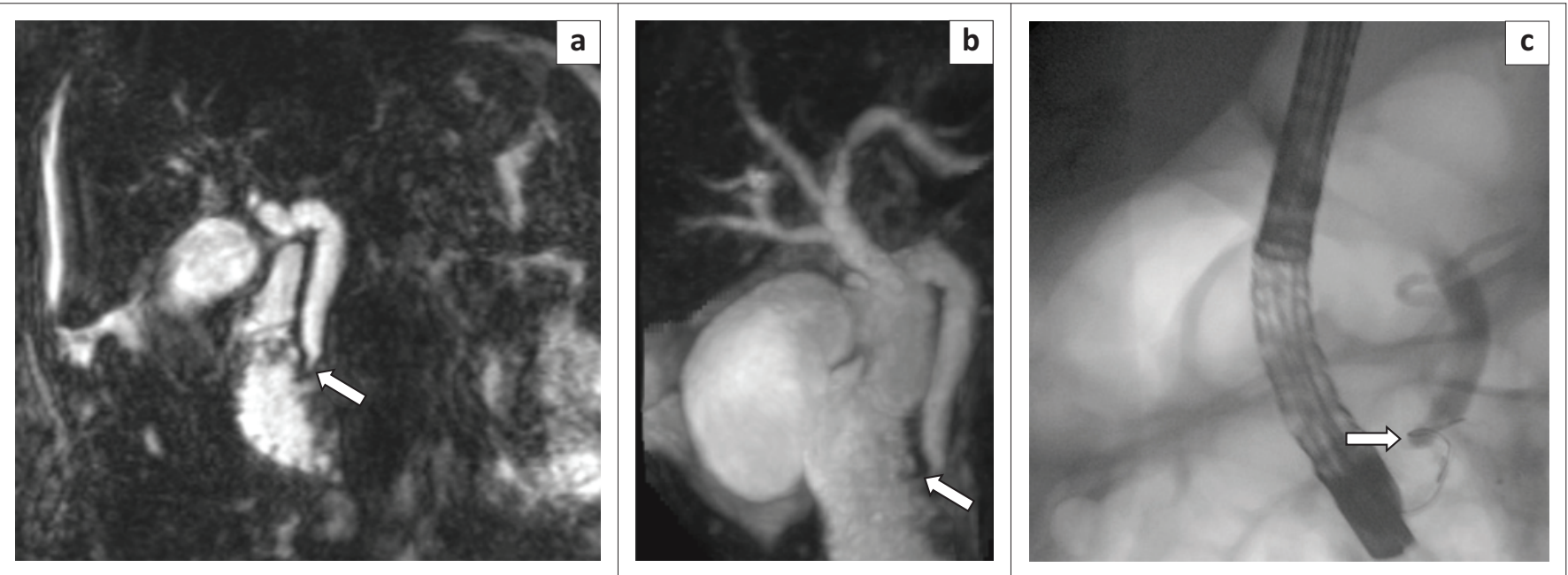

MRCP, magnetic resonance cholangiopancreatography; ERCP, endoscopic retrograde cholangiopancreatography

FIGURE 3: Eighty-seven-year-old woman with a benign stricture (white arrow) which was demonstrated by MRCP (a), 3D reformatted image from MRCP (b), and ERCP (c).

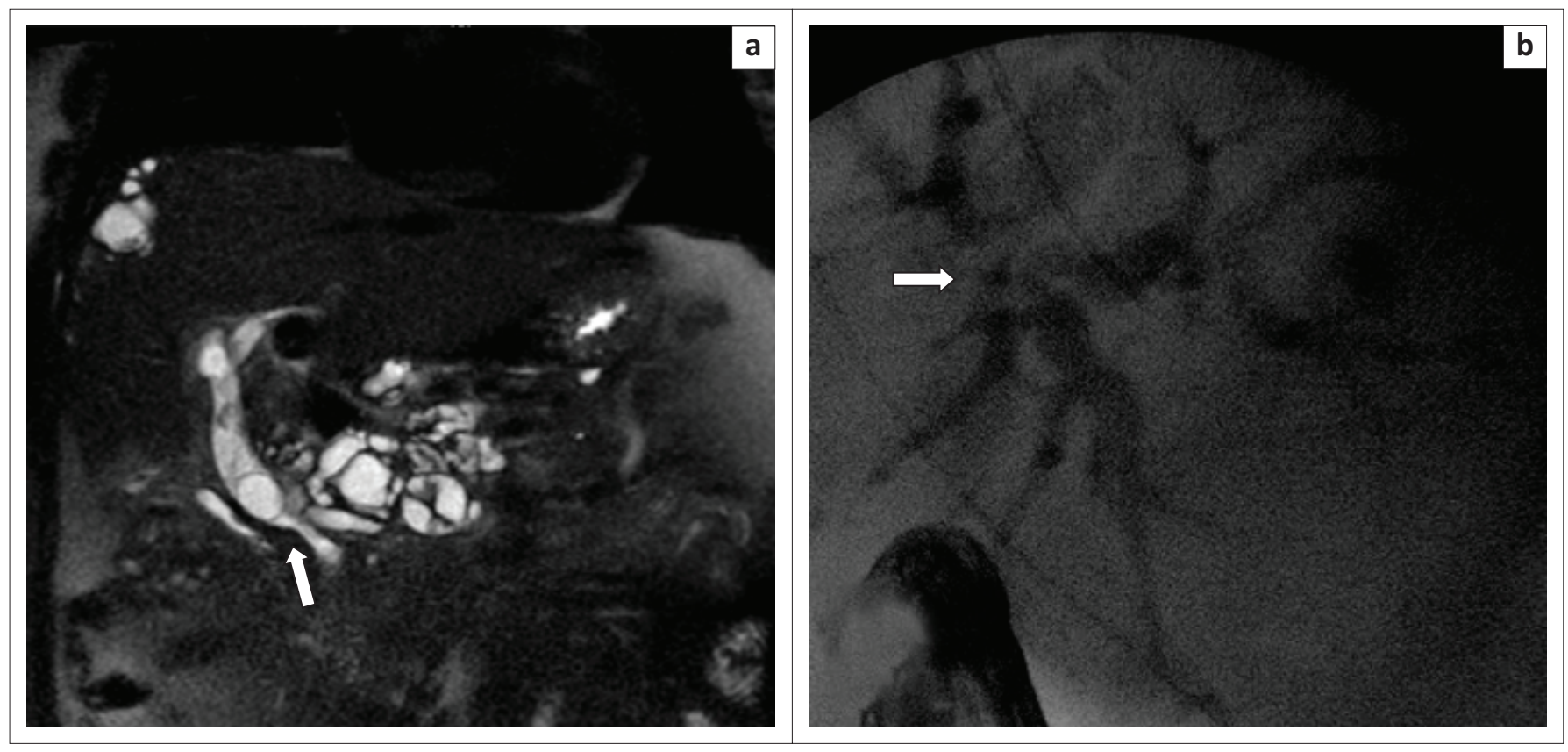

MRCP, magnetic resonance cholangiopancreatography; ERCP, endoscopic retrograde cholangiopancreatography.

FIGURE 4: Seventy-four-year-old woman with hydatid cyst and daughter vesicles: (a) The daughter cyst (arrow) inside the common bile duct is shown by MRCP; (b) irregular dilatations and compression of the biliary system owing to hydatid cysts (arrow) are seen by ERCP. 
TABLE 3: The statistical results of magnetic resonance cholangiopancreatography findings according to endoscopic retrograde cholangiopancreatography results as the gold standard.

\begin{tabular}{|c|c|c|c|c|c|c|c|c|c|}
\hline \multirow[t]{2}{*}{ Biliary disorders } & \multicolumn{2}{|c|}{ Sensitivity } & \multicolumn{2}{|c|}{ Specificity } & \multicolumn{2}{|r|}{ PPV } & \multicolumn{2}{|r|}{ NPV } & \multirow[t]{2}{*}{ Accuracy $(\%)$} \\
\hline & $\%$ & $(95 \% \mathrm{Cl})$ & $\%$ & $(95 \% \mathrm{Cl})$ & $\%$ & $(95 \% \mathrm{Cl})$ & $\%$ & $(95 \% \mathrm{Cl})$ & \\
\hline Stones & 100 & $94.31-100$ & 94.7 & $85.38-98.90$ & 95.4 & $87.47-98.44$ & 100 & $93.35-100$ & 97.5 \\
\hline Malignant tumour & 100 & $81.47-100$ & 100 & $96.34-100$ & 100 & $82.41-100$ & 100 & $96.26-100$ & 100 \\
\hline Benign stricture & 93.7 & $69.77-99.84$ & 100 & $96.45-100$ & 100 & $79.61-100$ & 99 & $93.86-99.85$ & 99 \\
\hline Ruptured hydatid cyst & 100 & $29.24-100$ & 100 & $96.82-100$ & 100 & $43.85-99.9$ & 100 & $96.74-100$ & 100 \\
\hline Normal & 88.2 & $63.56-98.54$ & 100 & $96.45-100$ & 100 & $79.61-100$ & 98 & $93.28-99.47$ & 98.2 \\
\hline
\end{tabular}

MRCP, magnetic resonance cholangiopancreatography; ERCP, endoscopic retrograde cholangiopancreatography; PPV, positive predictive value; NPV, negative predictive value; Cl, confidence interval.

of choledocholithiasis was lower than ours, at $95 \%$, but the specificity of MRCP was higher than ours, at $97 \%$. In our study, despite the high number of subcentimeter stones (30 out of 63), the specificity of MRCP was $94.7 \%$. Ito et al.'s MRCP accuracy was similar to ours at $97 \%$. Additionally, the accuracy of MRCP in differentiating benign strictures from malignant tumours was higher in our study (99\% v. 100\%).

Varghese et al. ${ }^{10}$ investigated 191 cases, comparing MRCP with ERCP, but most had normal findings. Only 31 cases with choledocholithiasis were included. Hekimoglu et al. ${ }^{1}$ investigated 269 cases, with 228 normal findings. The present study comprised a higher number of patients $(N=117)$ with various disorders $(n=100)$. There were only 17 patients with normal findings in our series. The number of our patients with choledocholithiasis $(n=63)$ was higher than that in similar studies in the literature. . $, 3,10,11,12^{2}$

In our series, 30 out of 63 patients had subcentimeter stones at ERCP. The specificity and PPV of MRCP in the diagnosis of calculi $<10 \mathrm{~mm}$ was $91 \%$ and $90 \%$, respectively. The specificity and PPV of MRCP for the diagnosis of stones $\geq 10 \mathrm{~mm}$ was $100 \%$. In a previous study, Zidi et al. ${ }^{3}$ found a lesser sensitivity (57.1\%) for MRCP in the detection of subcentimeter stones than in our study, where we found higher ratios of specificity and PPV at MRCP in the diagnosis of stones $\geq 10 \mathrm{~mm}$. Nandalur et al. ${ }^{11}$ also showed increased sensitivity in stones $>3 \mathrm{~mm}(94 \%-100 \%)$.

To the best of our knowledge, the effect of the number of stones in the detection of choledocholithiasis at MRCP has not been investigated before. Amongst solitary stones $(n=37)$ at ERCP, there were two false negative results for solitary stone at MRCP. Amongst patients with multiple stones $(n=26)$, there were five false positive results for patients with multiple stones owing to underestimating the number of solitary stones $(n=2)$ or misdiagnosis of subcentimeter stones $(n=5)$ at MRCP.

DWI is based on the random motion of water molecules in tissue. The degree of restriction to water molecule diffusion is directly associated with tissue cellularity and cell membrane integrity. Tumours with increased cellularity show more restricted movement of water molecules than in less cellular tumours. The degree of restricted diffusion is proportional to the degree of signal intensity in DWI. DWI should always be confirmed with an ADC map. The ADC map is a quantitative parameter calculated from DWI. Higher signal intensities in DWI and lower ADC values are defined as the result of restricted diffusion. On the other hand, higher ADC values indicate less cellularity of lesions. ${ }^{13,14}$

DWI is not a routine sequence used for the diagnosis of choledocholithiasis, but it is easily utilized because of the short scan time. At lower $b$ values, the biliary system will be hyperintense, similar to MRCP, and stones can easily be seen. WhetherDWI contributes to the diagnosis of choledocholithasis has not been investigated before. The sensitivity of DWI for stones $<10 \mathrm{~mm}$ and $\geq 10 \mathrm{~mm}$ was estimated to be $56.6 \%$ and $94.4 \%$, respectively. DWI was less sensitive to subcentimeter stones than MRCP. DWI is more sensitive to motion artifacts, which were common in our series, owing to difficulty in breath holding amongst elderly patients. The lower sensitivity of DWI in the diagnosis of choledocholithiasis was thought to be because of these artifacts.

MRCP was also found to be effective in differentiating benign strictures $(n=15)$ from tumours $(n=18)$. The sensitivity and specificity of MRCP was $100 \%$. These results were higher than the findings of other studies in the differential diagnosis of benign and malignant strictures. ${ }^{9,15}$ In our study, coronal and axial T2-weighted sequences and MRCP images were evaluated together; this could be the reason for the higher accuracy in our study.

The findings of previous studies on DWI and MRCP in diagnosis of tumours were similar to ours. ${ }^{13,14}$ In our study, all tumours were diagnosed with DWI or MRCP with $100 \%$ sensitivity and specificity. All malignant tumours showed diffusion restriction.

Despite the small number of hydatid cysts communicating with the biliary system, the localisation of biliary rupture, anatomical variations of the biliary system, and daughter cysts inside the biliary system were accurately shown on MRCP.

\section{Study limitations}

Our study has several limitations. Being a retrospective study, ERCP was not performed immediately after MRCP in all patients. The subcentimeter stones could have passed out spontaneously after MRCP; this could be a reason for false positive results at MRCP. ERCP is accepted as the gold standard but it is limited, especially in the diagnosis of subcentimeter stones and evaluating the intrahepatic biliary tree.

\section{Conclusion}

MRCP is a non-invasive, alternative imaging modality to ERCP for the evaluation of various pancreaticobiliary 
disorders, especially calculi $\geq 10 \mathrm{~mm}$, tumours, strictures and complicated hydatid cysts with biliary communication. DWI can be helpful in the diagnosis of choledocholithiasis and tumours. ERCP remains the modality of choice where intervention for the alleviation of cholestasis is required.

\section{Acknowledgements Competing interests}

The authors declare that they have no financial or personal relationships that may have inappropriately influenced them in writing this article.

\section{Authors' contributions}

Z.C.A. and F.K. were the project leaders; Z.C.A. and F.K. were responsible for project design and data collection; Z.C.A., F.K. and A.S.O. made conceptual contributions; Z.C.A., F.K., A.S.O., Y.B. and K.O. were responsible for analysis and/or interpretation; Z.C.A., F.K. and A.S.O. wrote the manuscript; and critical reviews were done by A.S.O., Y.B. and K.O.

\section{References}

1. Hekimoglu K, Ustundag Y, Dusak A, et al. MRCP vs. ERCP in the evaluation of biliary pathologies: Review of current literature. J Dig Dis. 2008;9(3):162-169. https:// doi.org/10.1111/j.1751-2980.2008.00339.x

2. Kaltenthaler EC, Walters SJ, Chilcott J, et al. MRCP compared to diagnostic ERCP for diagnosis when biliary obstruction is suspected: A systematic review. BMC Med Imaging. 2006;6:9.
3. Zidi SH, Prat F, Le Guen O, et al. Use of magnetic resonance cholangiography in the diagnosis of choledocholithiasis: Prospective comparison with a reference imaging method. Gut. 1999;44(1):118-122. https://doi.org/10.1136/gut.44.1.118

4. Hurter D, De Vries $C$, Potgieter $P$, et al. Accuracy of MRCP compared to ERCP in the diagnosis of bile duct disorders. SA J Radiol. 2008;12(1):14-22. https://doi. org/10.4102/sajr.v12i1.580

5. Wallner BK, Schumacher KA, Weidenmaier W, et al. Dilated biliary tract: Evaluation with MR cholangiography with a T2-weighted contrast-enhanced fast sequence. Radiology. 1991;181:805-808. https://doi.org/10.1148/radiology.181.3.1947101

6. Kaltenthaler E, Bravo Vergel Y, Chilcott J, et al. A systematic review and economic evaluation of magnetic resonance cholangiopancreatography compared with diagnostic endoscopic retrograde cholangiopancreatography. Health Techno Assess. 2004;8(10):1-102. https://doi.org/10.3310/hta8100

7. Costi R, Gnocchi A, Di Mario F, et al. Diagnosis and management of choledocholithiasis in the golden age of imaging, endoscopy and laparoscopy. World J Gastroenterol. 2014;20(37):13382-13401. https://doi.org/10.3748/wjg.v20.i37.13382

8. Griffin N, Charles-Edwards G, Grant LA. Magnetic resonance cholangiopancreatography: The ABC of MRCP. Insights Imaging. 2012;3(1):11-21. https://doi.org/10.1007/ s13244-011-0129-9

9. Ito K, Fujita N, Noda $Y$, et al. Clinical efficacy of magnetic resonance cholangiopancreatography in the diagnosis of biliary tract diseases. Nippon Shokakibyo Gakkai Zasshi. 2001;98(10):1164-1173.

10. Varghese JC, Liddell RP, Farrell MA, et al. Diagnostic accuracy of magnetic resonance cholangiopancreatography and ultrasound compared with direct cholangiography in the detection of choledocholithiasis. Clin Radiol. 2000; 55(1):25-35. https://doi.org/10.1053/crad.1999.0319

11. Nandalur KR, Hussain HK, Weadock WJ, et al. Possible biliary disease: Diagnostic performance of high-spatial resolution isotropic 3D T2-weighted MRCP. Radiology. 2008;249(3):883-890. https://doi.org/10.1148/radiol.2493080389

12. Mandelia A, Gupta AK, Verma DK, et al. The value of magnetic resonance cholangiopancreatography in the detection of choledocholithiasis. West Afr J Radiol. 2013;20:23-29. https://doi.org/10.4103/1115-1474.117907

13. Huang WC, Sheng J, Chen SY, et al. Differentiation between pancreatic carcinoma and mass-forming chronic pancreatitis: Usefulness of highbvalue diffusionweighted imaging. J Dig Dis. 2011;12:401-408. https://doi.org/10.1111/j.1751 2980.2011.00517.x

14. Cui $X Y$, Chen HW. Role of diffusion-weighted magnetic resonance imaging in the diagnosis of extrahepatic cholangiocarcinoma. World J Gastroenterol. 2010;16(25):3196. https://doi.org/10.3748/wjg.v16.i25.3196

15. Park MS, Kim TK, Kim KW, et al. Differentiation of extrahepatic bile duct cholangiocarcinoma from benign stricture: Findings at MRCP versus ERCP. Radiology. 2004;233:234-240. https://doi.org/10.1148/radiol.2331031446 